\title{
The First Principle Study of $\beta$-CuAgSe Subcells
}

\author{
Xiaoling Zhu, Chenglong Shi*, Zhipeng Shao \\ College of Science, University of Shanghai for Science and Technology, Shanghai, China \\ Email:182282008@st.usst.edu.cn, `brucetj@163.com,182282023@st.usst.edu.cn
}

How to cite this paper: Zhu, X.L., Shi, C.L. and Shao, Z.P. (2021) The First Principle Study of $\beta$-CuAgSe Subcells. Journal of Applied Mathematics and Physics, 9, 1549-1559. https://doi.org/10.4236/jamp.2021.97106

Received: June 22, 2021

Accepted: July 20, 2021

Published: July 23, 2021

Copyright $\odot 2021$ by author(s) and Scientific Research Publishing Inc. This work is licensed under the Creative Commons Attribution International License (CC BY 4.0).

http://creativecommons.org/licenses/by/4.0/ (c) (i) Open Access

\begin{abstract}
$\mathrm{CuAgSe}$ has been considered as a promising thermoelectric material because of its high mobility and low thermal conductivity. The superior performance of $\mathrm{CuAgSe}$ is closely related to its crystal structure and electric properties. In this work, the stabilities and electronic structures of different three $\mathrm{CuAgSe}$ subcells have been theoretically investigated using Vienna Ab initio Simulation Package (VASP) with DFT calculations. We found that the different occupations of copper atoms would affect the stability and electronic structures of $\mathrm{CuAgSe}$ subcells. The various directions of $\mathrm{Cu}$-Se chain in neighbor layers will result in different stabilities and electronic properties.
\end{abstract}

\section{Keywords}

CuAgSe, Subcells, Stability, Electronic Property

\section{Introduction}

Copper chalcogenides have been regarded as promising functional materials for their application in thermoelectrics [1] [2] [3], photovoltaics [4] [5], superconductivity [6] [7], sensors [8] and solar cell applications [9] [10] [11]. Among these copper chalcogenides, CuAgSe is a promising thermoelectric material because it has extremely high carrier mobility and relatively low thermal conductivity [12] [13] [14].

The superior performance of $\mathrm{CuAgSe}$ is closely related to its crystal structure and electric properties [15]. It had been reported that $\mathrm{CuAgSe}$ has two phases: low temperature $\beta$-phase and high temperature $\alpha$-phase, which is similar to $\mathrm{Cu}_{2} \mathrm{Se}$ [16] [17]. At low temperature, $\mathrm{CuAgSe}$ has a tetragonal or orthorhombic unit cell (with little difference of lattice parameter in b axis, listed in Table 1). Both of these two symmetries had been found in experimental works [18] [19]. In a unit cell of LT structure, two Ag atoms located in positions: $(1 / 4,1 / 4,0.551)$, $(3 / 4,3 / 4,0.449)$ and two Se atoms in positions: $(1 / 4,1 / 4,0.127),(3 / 4,3 / 4,0.873)$, 
respectively. Besides, there are four equivalent positions for two $\mathrm{Cu}$ atoms, namely $(1 / 4,3 / 4,0.105),(3 / 4,1 / 4,0.895),(3 / 4,1 / 4,0.105)$ and $(1 / 4,3 / 4,0.895)$.

Typically, $\mathrm{Cu}$ atoms will occupy the two positions in diagonal positions in a unit cell [20]. That is to say, one $\mathrm{Cu}$ atom in position: $(1 / 4,3 / 4,0.105)$ and another in position: $(3 / 4,1 / 4,0.895)$. In this case, chains will be formed by $\mathrm{Cu}$ atoms and their nearest Se atoms. As shown in Figure 1(a), these chains have the same direction and are connected as CuSe layers. Ag atoms take the places between $\mathrm{CuSe}$ layers to form a layered structure of $\beta$-CuAgSe (This subcell structure will be called S1 for short below).

Moreover, the equivalent positions for $\mathrm{Cu}$ atoms are not fully occupied, there are many vacancies for $\mathrm{Cu}$ to diffuse. In this way, $\mathrm{Cu}$ atoms can diffuse between the nearest $\mathrm{Cu}$-Se layers to form other two sublattice structures. In one subcell, $\mathrm{Cu}$ atoms will locate at the positions $(1 / 4,3 / 4,0.105)$ and $(3 / 4,1 / 4,0.105)$, as shown in Figure 1(b). In this structure, $\mathrm{Cu}$ atoms will get together in the same $\mathrm{Cu}-\mathrm{Se}$ layers. In their neighbor layers, there are only Se atoms (This subcell structure will be called S2 for short below). The third subcell structure contains two $\mathrm{Cu}$ atoms in positions $(1 / 4,3 / 4,0.105)$ and $(1 / 4,3 / 4,0.895)$. In this case, $\mathrm{Cu}$ atoms will also be chained with their nearest Se atoms. Different from the subcell above, these $\mathrm{Cu}$-Se chains have different directions with their neighbor layers, as shown in Figure 1(c) (This subcell structure will be called S3 for short below).

In this work, we will firstly discuss total energies of the mentioned low temperature $\mathrm{CuAgSe}$ subcells, to have a theoretical understanding of the stabilities of them. And then, the electronic band structures and projected density of states will also be discussed. Besides, DFT + U works are included to reveal the contributions from d electrons as well.

Table 1. The lattice parameters of orthorhombic structure and tetragonal structure of $\beta$-CuAgSe.

\begin{tabular}{ccccc}
\hline & & \multicolumn{3}{c}{ Lattice Parameter $(\AA)$} \\
Structure & Space Group & $\mathbf{a}$ & $\mathbf{b}$ & $\mathbf{c}$ \\
\cline { 3 - 4 } orthorhombic & Pmmn & 4.105 & 4.07 & 6.31 \\
tetragonal & $\mathrm{P} 4 / \mathrm{nmm}$ & 4.105 & 4.105 & 6.31 \\
\hline
\end{tabular}

a, b, c are the lattice parameters of a unitcell in three directions. The directions can be seen in Figure 1.
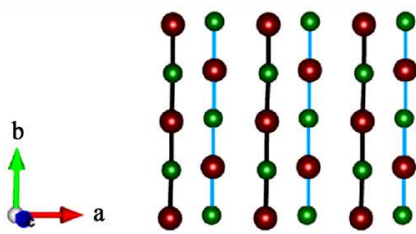

(a)

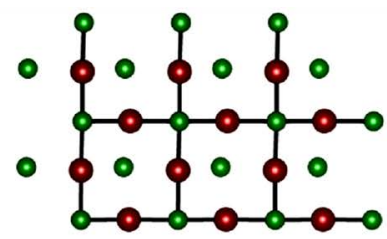

(b)

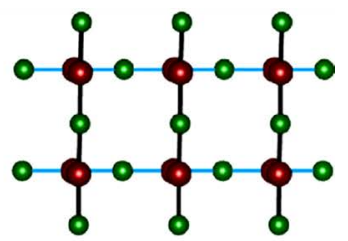

(c)

Figure 1. The top view of (a) S1, (b) S2 and (c) S3. Green, silvery and deep red spheres are respectively $\mathrm{Se}, \mathrm{Ag}$ and $\mathrm{Cu}$ atoms. The occupancy of the $\mathrm{Cu}$ site is 0.5. The crystal structures are visualized in VESTA [21]. For convenient observation, $\mathrm{Cu}$-Se bond in different layers is colored with color darkgray and color blue, respectively. 


\section{Computational Details}

The calculation of this work is based on density functional theory (DFT) [22] and implemented in the Vienna Ab initio Simulation Package (VASP) [23] [24]. We use the Perdew-Burke-Ernzerhof (PBE) type of generalized gradient approximation (GGA) as the exchange-correlation functional [25] [26] [27]. The valence electron configurations employed in our calculations are $\mathrm{Cu}(3 \mathrm{~d} 10,4 \mathrm{~s} 1)$, $\mathrm{Ag}(4 \mathrm{~d} 10,5 \mathrm{~s} 1)$ and $\mathrm{Se}(4 \mathrm{~s} 2,4 \mathrm{p} 4)$. The interaction between the core electrons and the valence electrons is included by the standard frozen-core projector augmented-wave (PAW) potentials provided within the VASP package [28] [29] [30]. The plane-wave cutoff energy is set to $600 \mathrm{eV}$ in this calculation. Because $\mathrm{Cu}$ atom and $\mathrm{Ag}$ atom belong to transition metal elements, in order to verify the results of electronic structures, we also use $\mathrm{PBE}+\mathrm{U}$ method to calculate the energy structures and density of states. The calculation use the PBE $+\mathrm{U}$ method with $\mathrm{U}=4.0 \mathrm{eV}$ to approximately describe the $\mathrm{Cu}-3 \mathrm{~d}$ electrons, and we also use $\mathrm{U}=2.0 \mathrm{eV}$ to approximately describe the Ag-4d electrons [31] [32] [33]. During the optimization, in consideration of dimensions of the structure and the convergence of the forces, we adopt the Monkhorst-Pack scheme k-point mesh [34] [35] from gamma to the $21 \times 21 \times 21$ point and use finer k-points to further calculate the electronic structures. The relaxation of the electronic degrees of freedom stops when both the total energy and the band structure energy variations between two steps are smaller than $10-6 \mathrm{eV}$. When we do a static calculation, we use ISMEAR $=-5$ and ionic relaxation goes on as long as any force is larger than $0.0001 \mathrm{eV} / \AA$, but it will immediately stop when the force is larger than $0.02 \mathrm{eV} / \AA$. The detailed atomic coordinates are listed in Table 2. We construct three subcell through VESTA to concretely show these three configurations (as shown in Figure 1).

\section{Results and Discussion}

Before reporting the electronic structures of the mentioned subcells, we will firstly discuss the stability of them. The stability of a material can be characterized by the total energy. The total energy, $E_{\text {tot }}$ can be expressed by Equation (1):

$$
E_{\text {tot }}=E_{\text {energy without entropy }}+T \times S
$$

where $E_{\text {tot }}$ is the total energy, $E_{\text {energy without entropy }}$ is the energy without entropy. In this work, $T \times S$ is set to be 0 , so that $E_{\text {tot }}$ is equal to $E_{\text {energy without entropy. Therefore, }}$ we calculated the total energies of three $\mathrm{CuAgSe}$ subcells with various lattice

Table 2. The detailed atomic coordinates of the three sub-lattices structures of CuAgSe $[20]$.

\begin{tabular}{|c|c|c|c|c|c|c|}
\hline Structure & Ag1 & $\mathrm{Ag} 2$ & Se1 & $\mathrm{Se} 2$ & $\mathrm{Cul}$ & $\mathrm{Cu} 2$ \\
\hline S1 & & & & & $(1 / 4,3 / 4,0.105)$ & $(3 / 4,1 / 4,0.895)$ \\
\hline S2 & $\begin{array}{c}(1 / 4,1 / 4 \\
0.551)\end{array}$ & $\begin{array}{c}(3 / 4,3 / 4 \\
0.449)\end{array}$ & $\begin{array}{c}(1 / 4,1 / 4 \\
0.127)\end{array}$ & $\begin{array}{c}(3 / 4,3 / 4 \\
0.873)\end{array}$ & $(1 / 4,3 / 4,0.105)$ & $(1 / 4,3 / 4,0.895)$ \\
\hline S3 & & & & & $(1 / 4,3 / 4,0.105)$ & $(3 / 4,1 / 4,0.105)$ \\
\hline
\end{tabular}


parameters, to determine the influence of copper occupation on the stabilities and electronic structures to all mentioned subcells above.

\subsection{The Stabilities}

The calculated total energies with various scaled lattice parameters have been shown in Figure 2. Where the scaled 1.00 refers to the lattice parameter reported in experimental works [20] [36]. Both orthorhombic and tetragonal structures are plotted together for convenient comparison. For these two symmetries, the relationship between total energies and scaled lattice parameters are very similar, the little differences in $b$-axis do not have significant effect on the total energies.

For S1 and S2, their total energy plots have very similar trends. Although there is a little difference in the plots of orthorhombic and tetragonal symmetries, they have the same lowest positions of the total energy, which located at 0.99 , very closely to former experimental works [37] [38]. As listed in Table 3, for S1 and $\mathrm{S} 2$, the lowest energies are $-23.0682 \mathrm{eV}$ (ortho) and $-23.0548 \mathrm{eV}$ (tetra) for S1 and $-23.0684 \mathrm{eV}$ (ortho) and $-23.0550 \mathrm{eV}$ (tetra) for S2, respectively. These locations of lowest total energies match well with former experimental works, which revealed the coexistence of these two symmetries [16] [39].

The total energy plot of S3 is quietly different from those of S1 and S2. As shown in Figure 2(c), the lines oscillate firstly and then go down to smaller values.

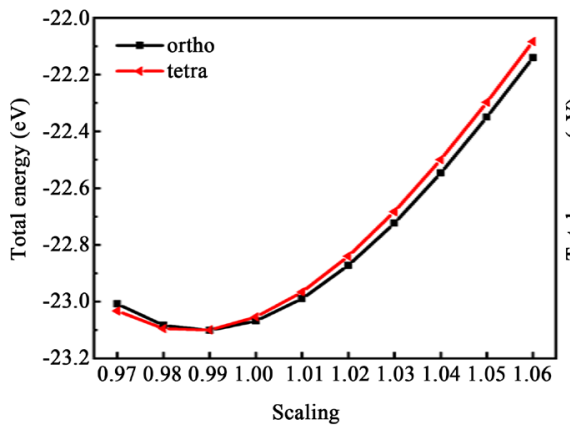

(a)

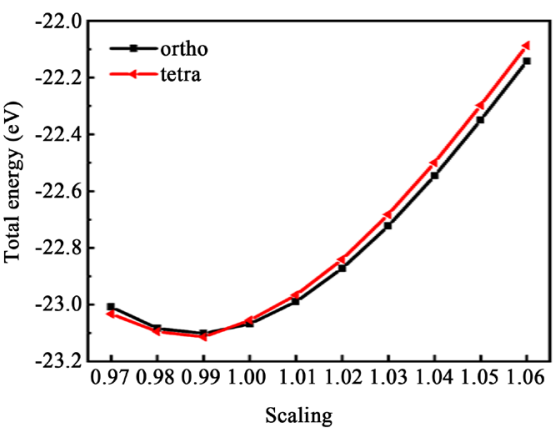

(b)

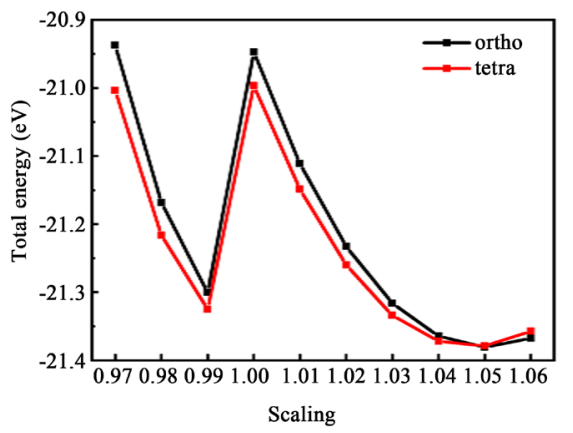

(c)

Figure 2. The total energies of S1, S2 and S3 with scaling the lattice parameters. (a)-(c) results using PBE recipe. From left to right are S1, S2 and S3, respectively. The black line represents orthogonal structure and the red line represents tetragonal structure.

Table 3. The total energy of orthorhombic and tetragonal phase of S1, S2, S3 at the equilibrium lattice constants in using PBE recipe.

\begin{tabular}{ccc}
\hline \multirow{2}{*}{ Structure } & Phase (space group) & Total Energy $(\mathrm{eV})$ \\
& & PBE \\
\hline \multirow{2}{*}{ S1 } & & -23.0682 \\
& & -23.0548 \\
S2 & Trthorhombic $(\mathrm{Pmmn})$ & -23.0684 \\
& & -23.0550 \\
Setragonal $(\mathrm{P} 4 / \mathrm{nmm})$ & -20.9473 \\
& & -20.9965
\end{tabular}


The lowest total energies located at 1.05 , which is a bit far from those in experimental works [38]. Besides, the lowest energy of S3 is much higher than those of S1 and S2. In the considerations of above, we think S3 is relatively unstable subcell.

\subsection{The Electronic Properties}

\subsubsection{Band Structures}

We first carry out the electronic band structures of S1, S2 and S3. For a direct comparison, tetragonal and orthorhombic structures are plotted together with the same path: $\Gamma-\mathrm{X}-\mathrm{M}-\Gamma-\mathrm{Z}[40]$, since they have nearly the same lattice constant (so the reciprocal lattice). It can be seen that the plotted bands for tetragonal and orthorhombic structures are almost overlapped, which means that the symmetry has little effect on the electronic structure, so our discussions will focus on the differences between different subcells.

In Figure 3(a), S1 is predicted to be a semimetal with the Fermi level crossing bands along $\Gamma-\mathrm{X}$ and $\Gamma-\mathrm{Z}$ lines. There is a peak at Fermi level in the total density of states for S1. While for S2 and S3 (shown in Figure 3(b) and Figure 3(c)), they are predicted to be semimetals as well (but smaller peak at Fermi level), according to the total density of states. Early experimental works [12] had reported $\mathrm{CuAgSe}$ is a semimetal. As mentioned above, the total energy of S1 and S2 are nearly the same. Besides, the total energy of S3 is relatively high. If so, S1 and S2 are more like to exist and contribute to the electronic properties of CuAgSe. That is to say, the semimetal behavior of CuAgSe may result from the contributions of both $\mathrm{S} 1$ and $\mathrm{S} 2$.

\subsubsection{DFT + U}

Recently, Råsander et al. studied the electronic properties of fluorite Cu2Se using the DFT $+\mathrm{U}$ approaches. The results obtained by LDA $+\mathrm{U}$ show that a adequately high $\mathrm{U}$ opens up a gap [41]. Moreover, $\mathrm{Yb}$ Zhang et al using the $\mathrm{mBJ}$ $+\mathrm{U}$ approach investigated $\mathrm{Cu} 2 \mathrm{X}(\mathrm{X}=\mathrm{S}, \mathrm{Se}, \mathrm{Te})$, and found impose different $\mathrm{U}$

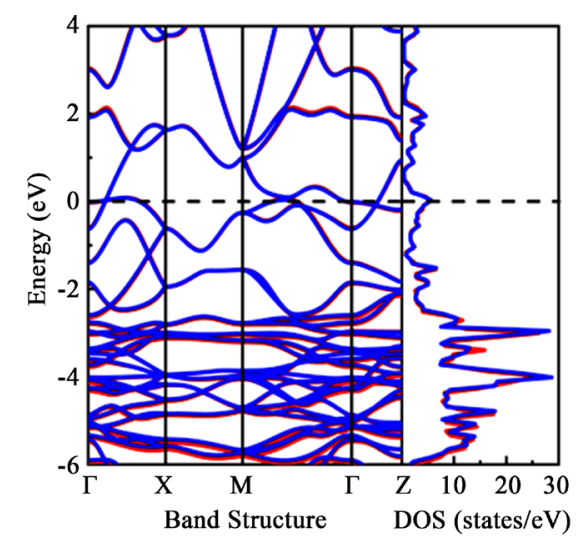

(a)

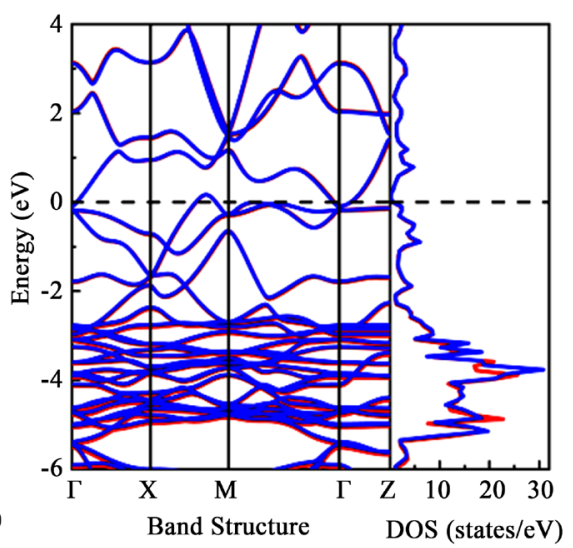

(b)

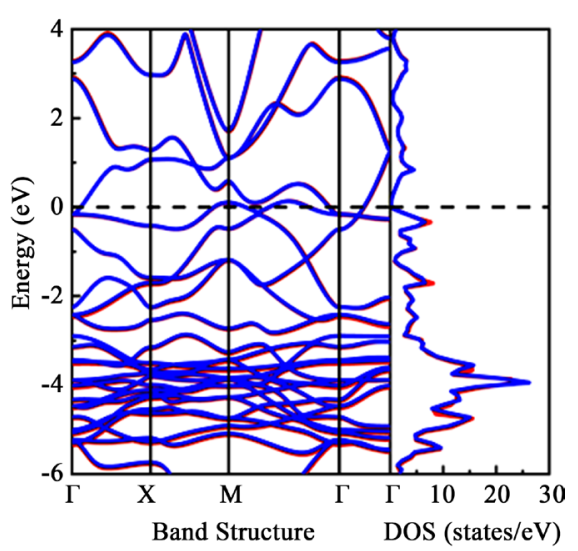

(c)

Figure 3. The band structures and total DOS of S1, S2, S3 under PBE. The red lines represent orthogonal structure and the blue lines are tetragonal structure. The dash lines are Fermi level. 
values on the $3 \mathrm{~d}$ orbital of $\mathrm{Cu}$, and the band gap gradually increases with increasing $U$ [33]. Hence, we introduced DFT $+U$ to further study the electronic band structures of $\mathrm{CuAgSe}$. We use the $\mathrm{PBE}+\mathrm{U}$ method with $\mathrm{U}=4.0 \mathrm{eV}$ to approximately describe the $\mathrm{Cu}-3 \mathrm{~d}$ electrons, and we also use $\mathrm{U}=2.0 \mathrm{eV}$ to approximately describe the Ag-4d electrons. The calculated band structures are shown in Figure 4, the plotted bands for tetragonal and orthorhombic structures are almost overlapped. Their band structures have little difference compared with them without $\mathrm{U}$ indicating $\mathrm{CuAgSe}$ in low temperature still is a metal conductor and the application of $\mathrm{U}$ has little effect on the electronic band structures of CuAgSe.

\subsubsection{Density of States}

Then we investigate the projected density of states of $\mathrm{CuAgSe}$ with different $\mathrm{Cu}$ positions. The calculated DOS of tetragonal subcells and orthorhombic subcells are very similar, so only the DOS of tetragonal subcells are shown in Figure 5.

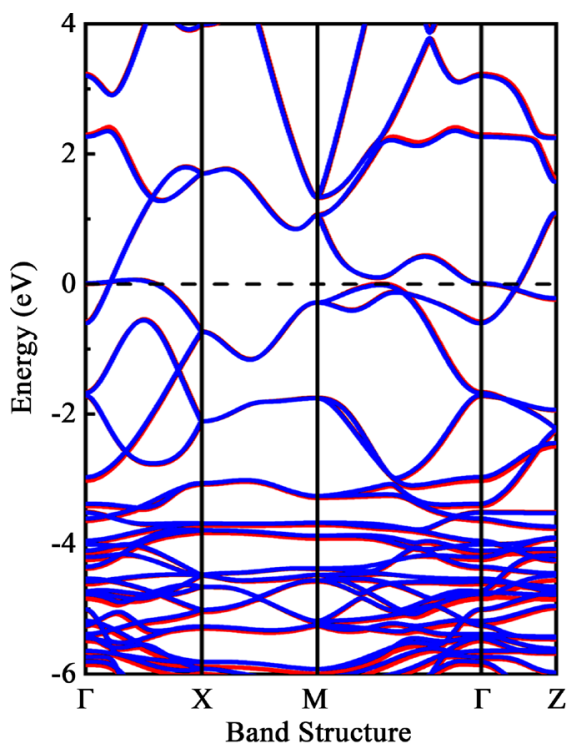

(a)

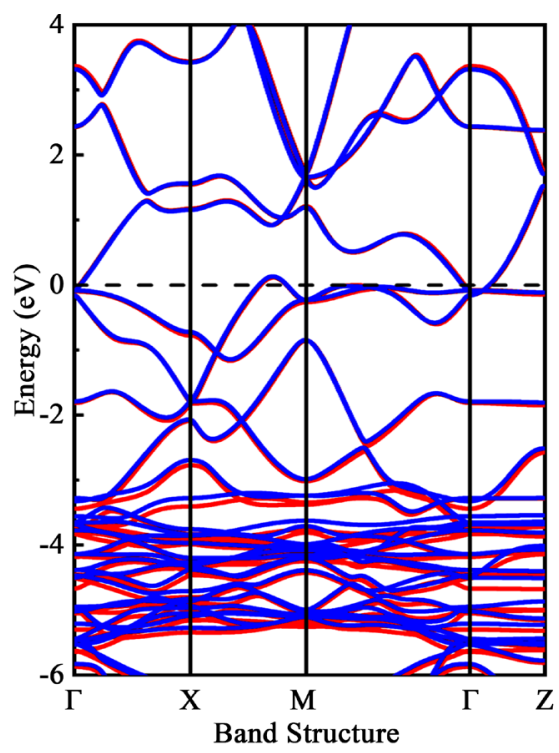

(b)

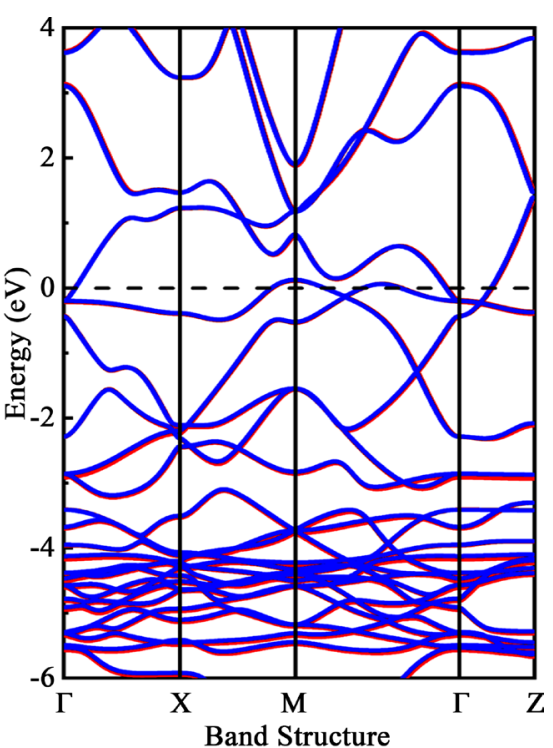

(c)

Figure 4. The Band structures of $\mathrm{S} 1, \mathrm{~S} 2$ and $\mathrm{S} 3$ with $\mathrm{DFT}+\mathrm{U}$ (we use $\mathrm{U}=4.0 \mathrm{eV}$ to approximately describe the $\mathrm{Cu}-3 \mathrm{~d}$ electrons, and we also use $\mathrm{U}=2.0 \mathrm{eV}$ to approximately describe the Ag-4d electrons.). The red lines represent the orthorhombic structure of S1, S2 and S3, the blue lines are the tetragonal structure of S1, S2 and S3.

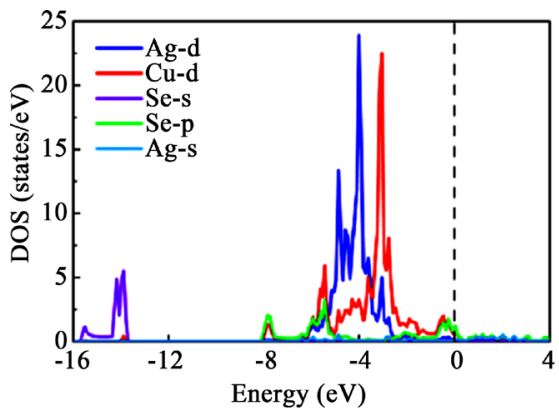

(a)

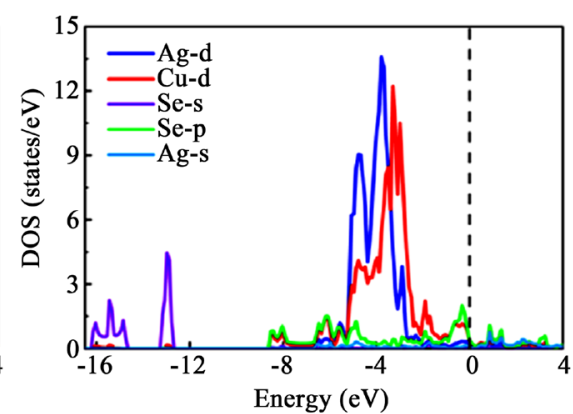

(b)

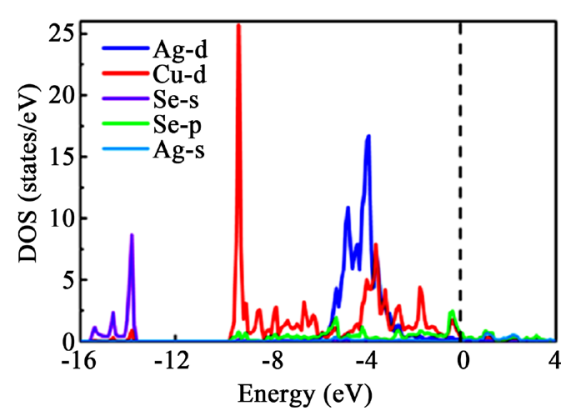

(c)

Figure 5. The DOS of tetragonal symmetry structures of S1, S2 and S3 with using PBE recipe, respectively. The dotted lines represent the Fermi levels. 
For S1 (Figure 5(a)), the lowest band located at $-16 \mathrm{eV}$ to $-13.5 \mathrm{eV}$ is derived from Se $4 \mathrm{~s}$ states and the semimetal behavior is mainly contributed by $\mathrm{Cu} 3 \mathrm{~d}$ electrons and Se $4 \mathrm{p}$ electrons. The hybridization of $\mathrm{Cu} 3 \mathrm{~d}$ electrons and $\mathrm{Se} 4 \mathrm{p}$ electrons can also be found in $-8 \mathrm{eV}$ to $-7.5 \mathrm{eV}$ and $-6 \mathrm{eV}$ to $-5 \mathrm{eV}$. Besides, comparable Ag $4 \mathrm{~d}$ states are found in $-6 \mathrm{eV}$ to $-5.3 \mathrm{eV}$ as well. The region from $-5 \mathrm{eV}$ to $-2.5 \mathrm{eV}$ is mainly built up by $\mathrm{Cu} 3 \mathrm{~d}$ states and $\mathrm{Ag} 4 \mathrm{~d}$ states. The $\mathrm{Cu} 3 \mathrm{~d}$ peak and Ag $4 \mathrm{~d}$ peak lie between the energy level discussed above. This indicates the hybridization below Fermi level are anti-bonding states and bonding states lie below $-5 \mathrm{eV}$, respectively.

The $\mathrm{Cu} 3 \mathrm{~d}$ and $\mathrm{Se} 4 \mathrm{p}$ hybridization of S2 is similar to S1. We can see in Figure 5(b), the bands of S2 located $-16.5 \mathrm{eV}$ to $-14.5 \mathrm{eV}$ and $-13.5 \mathrm{eV}$ to $-12.5 \mathrm{eV}$ are also derived from Se 4 s states. Nevertheless, the mixing states of $\mathrm{Cu} 3 \mathrm{~d}$ and $\mathrm{Se} 4 \mathrm{p}$ lie at lower energy level, just below the Fermi level. For S2, the overlap of Ag 4d states and $\mathrm{Cu} 3 \mathrm{~d}$ states from $-5 \mathrm{eV}$ to $-3 \mathrm{eV}$ is much larger than that of $\mathrm{S} 1$, indicate a stronger correlation of $\mathrm{Cu} 3 \mathrm{~d}$ electrons and $\mathrm{Ag} 4 \mathrm{~d}$ electrons.

The calculated DOS of S3 (as shown in Figure 5(c)) is very different from S1 and S2. Between $-16 \mathrm{eV}$ and $-13.5 \mathrm{eV}$, the lowest band is contributed by Se $4 \mathrm{~s}$ states, and S3 has higher states than the other two structures. The pd hybridization of $\mathrm{Cu} 3 \mathrm{~d}$ electron and $\mathrm{Se} 4 \mathrm{p}$ electron becomes much weaker. Moreover, at the region from $-10 \mathrm{eV}$ to $-6 \mathrm{eV}$, the DOS is almost composed by $\mathrm{Cu} 3 \mathrm{~d}$ states, in contrast to $\mathrm{S} 1$ and $\mathrm{S} 2$, there are small $\mathrm{Cu} 3 \mathrm{~d}$ peaks refer to the pd hybridization. Comparable $\mathrm{Cu} 3 \mathrm{~d}$ states and $\mathrm{Ag} 4 \mathrm{~d}$ states are found in $-3.5 \mathrm{eV}$ to $-3 \mathrm{eV}$, indicate a correlation of them.

As discussed above, S1 and S2 have nearly the same total energies. The difference of their crystal structures is attributed to the directions of $\mathrm{Cu}-\mathrm{Se}$ chains. The $\mathrm{Cu}-\mathrm{Se}$ chains are parallel in $\mathrm{S} 1$ and perpendicular in $\mathrm{S} 2$, respectively. Because of the layered structure, the bonding states are basically composed by $\mathrm{Cu}$-Se layers. Thus, the differences of electronic structures between S1, S2, and even S3, are contributed by the pd hybridizations of $\mathrm{Cu}$ and Se. Since the total energy of S3 is much higher than S1 and S2, the observed behavior of CuAgSe may contributed from a mixing of the feature of S1 and S2, i.e., the directions of Cu-Se chains.

\section{Conclusion}

In this work, the total energies, electronic structures and density of states of three different $\mathrm{CuAgSe}$ subcells have been systematically studied by first principle calculations. Tetragonal and orthorhombic CuAgSe has nearly the same total energies, for both S1 and S2. The observed results that located at 0.99 S1 and S2 have the lowest total energies matched well with former experimental works. However, S3 has its lowest total energy located at 1.05, which is a bit far from experimental results. The calculated electronic structures show that all the three CuAgSe subcells are semimetals, and the little differences in b-axis do not have significant effect on the total energies and electronic properties. Moreover, DFT 
$+\mathrm{U}$ works were adopted to reveal the contributions from $\mathrm{d}$ electrons as well, but there was little effect on the electronic band structures. The projected density of states shows that the semimetal behavior of $\beta$-CuAgSe is mainly contributed by $\mathrm{Cu} 3 \mathrm{~d}$ electrons and Se $4 \mathrm{p}$ electrons, and the differences of electronic structures between S1, S2, and even S3, are contributed by the pd hybridizations of $\mathrm{Cu}$ and Se. The observed electronic structures and behaviors of CuAgSe may be attributed from the different $\mathrm{Cu}$-Se chain directions. Study on the layered structures and subcells will help understand copper chalcogenides with $\mathrm{Cu}$ vacancies [42] [43].

\section{Acknowledgements}

This work is supported by the National Natural Science Foundation of China Grants (21905715).

\section{Conflicts of Interest}

The authors declare no conflicts of interest regarding the publication of this paper.

\section{References}

[1] Zheng, X.F., Liu, C.X., Yan, Y.Y. and Wang, Q. (2014) A Review of Thermoelectrics Research-Recent Developments and Potentials for Sustainable and Renewable Energy Applications. Renewable and Sustainable Energy Reviews, 32, 486-503. https://doi.org/10.1016/j.rser.2013.12.053

[2] Moroz, N.A., Olvera, A., Willis, G.M. and Poudeu, P.F. (2015) Rapid Direct Conversion of $\mathrm{Cu}(2-\mathrm{x}) \mathrm{Se}$ to $\mathrm{CuAgSe}$ Nanoplatelets via Ion Exchange Reactions at Room Temperature. Nanoscale, 7, 9452-9456. https://doi.org/10.1039/C5NR01451D

[3] Wei, T.-R., Qin, Y., Deng, T., Song, Q., Jiang, B., Liu, R., Qiu, P., Shi, X. and Chen, L. (2019) Copper Chalcogenide Thermoelectric Materials. Science China Materials, 62, 8-24. https://doi.org/10.1007/s40843-018-9314-5

[4] Jackson, P., Hariskos, D., Lotter, E., Paetel, S., Wuerz, R., Menner, R., Wischmann, W. and Powalla, M. (2011) New World Record Efficiency for Cu(In,Ga)Se2 Thin-Film Solar Cells beyond 20\%. Progress in Photovoltaics. Research and Applications, 19, 894-897. https://doi.org/10.1002/pip.1078

[5] Gahlot, S., Dappozze, F., Singh, D., Ahuja, R., Cardenas, L., Burel, L., Amans, D., Guillard, C. and Mishra, S. (2020) Room-Temperature Conversion of $\mathrm{Cu}_{2-\mathrm{x}} \mathrm{Se}$ to CuAgSe Nanoparticles to Enhance the Photocatalytic Performance of Their Composites with $\mathrm{TiO}_{2}$. Dalton Transactions, 49, 3580-3591. https://doi.org/10.1039/C9DT04726C

[6] Das, T. and Balatsky, A.V. (2011) Modulated Superconductivity Due to Vacancy and Magnetic Order in $\mathrm{A}_{\mathrm{y}} \mathrm{Fe}_{2-\mathrm{x} / 2} \mathrm{Se}_{2}[\mathrm{~A}=\mathrm{Cs}, \mathrm{K},(\mathrm{Tl}, \mathrm{Rb}),(\mathrm{Tl}, \mathrm{K})]$ Iron-Selenide $\mathrm{Su}$ perconductors. Physical Review B, 84, Article ID: 115117. https://doi.org/10.1103/PhysRevB.84.115117

[7] Dagotto, E. (2013) Colloquium: The Unexpected Properties of Alkali Metal Iron Selenide Superconductors. Reviews of Modern Physics, 85, 849-867. https://doi.org/10.1103/RevModPhys.85.849

[8] Hotzel, G. and Weppner, W. (1986) Application of Fast Ionic Conductors in Solid 
State Galvanic Cells for Gas Sensors. Solid State Ionics, 18-19, 1223-1227. https://doi.org/10.1016/0167-2738(86)90338-3

[9] Okimura, H. and Matsumae, T. (1980) Electrical Properties of $\mathrm{Cu}_{2-\mathrm{x}} \mathrm{Se}$ Thin Films and Their Application for Solar Cells. Thin Solid Films, 71, 53-59.

https://doi.org/10.1016/0040-6090(80)90183-2

[10] Nguyen, M.C., Choi, J.-H., Zhao, X., Wang, C.-Z., Zhang, Z. and Ho, K.-M. (2013) New Layered Structures of Cuprous Chalcogenides as Thin Film Solar Cell Materials: $\mathrm{Cu}_{2} \mathrm{Te}$ and $\mathrm{Cu}_{2} \mathrm{Se}$. Physical Review Letters, 111, Article ID: 165502. https://doi.org/10.1103/PhysRevLett.111.165502

[11] Todorov, T.K., Tang, J., Bag, S., Gunawan, O., Gokmen, T., Zhu, Y. and Mitzi, D.B. (2013) Beyond 11\% Efficiency: Characteristics of State-of-the-Art $\mathrm{Cu}_{2} \mathrm{ZnSn}(\mathrm{S}, \mathrm{Se})_{4}$ Solar Cells. Advanced Energy Materials, 3, 34-38. https://doi.org/10.1002/aenm.201200348

[12] Ishiwata, S., Shiomi, Y., Lee, J.S., Bahramy, M.S., Suzuki, T., Uchida, M., Arita, R., Taguchi, Y. and Tokura, Y. (2013) Extremely High Electron Mobility in a Phonon-Glass Semimetal. Natural Materials, 12, 512-517.

https://doi.org/10.1038/nmat3621

[13] Han, C., Sun, Q., Cheng, Z.X., Wang, J.L., Li, Z., Lu, G.Q. and Dou, S.X. (2014) Ambient Scalable Synthesis of Surfactant-Free Thermoelectric CuAgSe Nanoparticles with Reversible Metallic-n-p Conductivity Transition. Journal of the American Chemical Society, 136, 17626-17633. https://doi.org/10.1021/ja510433j

[14] Qiu, P.F., Wang, X.B., Zhang, T.S., Shi, X. and Chen, L.D. (2015) Thermoelectric Properties of Te-Doped Ternary CuAgSe Compounds. Journal of Materials Chemistry A, 3, 22454-22461. https://doi.org/10.1039/C5TA06780D

[15] Qiu, W., Lu, P., Yuan, X., Xu, F., Wu, L., Ke, X., Liu, H., Yang, J., Shi, X., Chen, L., Yang, J. and Zhang, W. (2016) Structure Family and Polymorphous Phase Transition in the Compounds with Soft Sublattice: $\mathrm{Cu}_{2} \mathrm{Se}$ as an Example. The Journal of Chemical Physics, 144, Article ID: 194502. https://doi.org/10.1063/1.4948609

[16] Hong, A.J., Li, L., Zhu, H.X., Zhou, X.H., He, Q.Y., Liu, W.S., Yan, Z.B., Liu, J.M. and Ren, Z.F. (2014) Anomalous Transport and Thermoelectric Performances of CuAgSe Compounds. Solid State Ionics, 261, 21-25.

https://doi.org/10.1016/j.ssi.2014.03.025

[17] Chrissafis, K., Vouroutzis, N., Paraskevopoulos, K.M., Frangis, N. and Manolikas, C. (2004) Phase Transformation in CuAgSe: A DSC and Electron Diffraction Examination. Journal of Alloys and Compounds, 385, 169-172.

https://doi.org/10.1016/j.jallcom.2004.04.119

[18] Shi, C., Xi, X., Hou, Z., Liu, E., Wang, W., Jin, S., Wu, Y. and Wu, G. (2016) Atomic-Level Characterization of Dynamics of Copper Ions in CuAgSe. The Journal of Physical Chemistry C, 120, 3229-3234. https://doi.org/10.1021/acs.jpcc.5b12296

[19] Wang, X., Qiu, P., Zhang, T., Ren, D., Wu, L., Shi, X., Yang, J. and Chen, L. (2015) Compound Defects and Thermoelectric Properties in Ternary CuAgSe-Based Materials. Journal of Materials Chemistry A, 3, 13662-13670.

https://doi.org/10.1039/C5TA02721G

[20] Frueh Jr., A.J., Czamanske, G.K. and Knight, C.H. (1956) The Ciystallography of Eucairite, CuAgSe. Zeitschrift fur KristaUographie, 108, 389-396. https://doi.org/10.1524/zkri.1957.108.5-6.389

[21] Momma, K. and Izumi, F. (2008) VESTA: A Three-Dimensional Visualization System for Electronic and Structural Analysis. Journal of Applied Crystallography, 41, 653-658. https://doi.org/10.1107/S0021889808012016 
[22] Kohn, W. and Sham, L.J. (1965) Self-Consistent Equations Including Exchange and Correlation Effects. Physical Review, 140, A1133-A1138. https://doi.org/10.1103/PhysRev.140.A1133

[23] Kresse, G. and Furthmüller, J. (1996) Efficient Iterative Schemes for Ab-Initio Total-Energy Calculations Using a Plane-Wave Basis Set. Physical Review B, 54, 11169-11186. https://doi.org/10.1103/PhysRevB.54.11169

[24] Kresse, G. and Furthmüller, J. (1996) Efficiency of Ab-Initio Total Energy Calculations for Metals and Semiconductors Using a Plane-Wave Basis Set. Computational Materials Science, 6, 15-50. https://doi.org/10.1016/0927-0256(96)00008-0

[25] Perdew, J.P., Burke, K. and Ernzerhof, M. (1996) Generalized Gradient Approximation Made Simple. Physical Review Letters, 77, 3865-3868.

https://doi.org/10.1103/PhysRevLett.77.3865

[26] Becke, A.D. (2014) Perspective: Fifty Years of Density-Functional Theory in Chemical Physics. The Journal of Chemical Physics, 140, 18A301. https://doi.org/10.1063/1.4869598

[27] Pedroza, L.S., da Silva, A.J.R. and Capelle, K. (2009) Gradient-Dependent Density Functionals of the Perdew-Burke-Ernzerhof Type for Atoms, Molecules, and Solids. Physical Review B, 79, 201106(R). https://doi.org/10.1103/PhysRevB.79.201106

[28] Blochl, P.E. (1994) Projector Augmented-Wave Method. Physical Review B, Condensed Matter, 50, 17953-17979. https://doi.org/10.1103/PhysRevB.50.17953

[29] Chu, W., Zheng, Q., Akimov, A.V., Zhao, J., Saidi, W.A. and Prezhdo, O.V. (2020) Accurate Computation of Nonadiabatic Coupling with Projector Augmented-Wave Pseudopotentials. The Journal of Physical Chemistry Letters, 11, 10073-10080. https://doi.org/10.1021/acs.jpclett.0c03080

[30] Kresse, G. and Joubert, D. (1999) From Ultrasoft Pseudopotentials to the Projector Augmented-Wave Method. Physical Review B, 59, 1758-1775.

https://doi.org/10.1103/PhysRevB.59.1758

[31] Sun, Y., Xi, L., Yang, J., Wu, L., Shi, X., Chen, L., Snyder, J., Yang, J. and Zhang, W. (2017) The "Electron Crystal" Behavior in Copper Chalcogenides $\mathrm{Cu}_{2} \mathrm{X}(\mathrm{X}=\mathrm{Se}, \mathrm{S})$. Journal of Materials Chemistry A, 5, 5098-5105. https://doi.org/10.1039/C6TA10725G

[32] Zhang, Y., Yuan, X., Sun, X., Shih, B.-C., Zhang, P. and Zhang, W. (2011) Comparative Study of Structural and Electronic Properties of Cu-Based Multinary Semiconductors. Physical Review B, 84, Article ID: 075127. https://doi.org/10.1103/PhysRevB.84.075127

[33] Zhang, Y., Wang, Y., Xi, L., Qiu, R., Shi, X., Zhang, P. and Zhang, W. (2014) Electronic Structure of Antifluorite $\mathrm{Cu}_{2} \mathrm{X}(\mathrm{X}=\mathrm{S}$, Se, Te) within the Modified Becke-Johnson Potential plus an On-Site Coulomb U. The Journal of Chemical Physics, 140, Article ID: 074702. https://doi.org/10.1063/1.4865257

[34] Froyen, S. (1989) Brillouin-Zone Integration by Fourier Quadrature: Special Points for Superlattice and Supercell Calculations. Physical Review B, Condensed Matter, 39, 3168-3172. https://doi.org/10.1103/PhysRevB.39.3168

[35] Monkhorst, H.J. and Pack, J.D. (1976) Special Points for Brillouin-Zone Integrations. Physical Review B, 13, 5188-5192. https://doi.org/10.1103/PhysRevB.13.5188

[36] Asadov, Y.G., Aliyev, Y.I. and Babaev, A.G. (2015) Polymorphic Transformations in $\mathrm{Cu}_{2} \mathrm{Se}, \mathrm{Ag}_{2} \mathrm{Se}, \mathrm{AgCuSe}$ and the Role of Partial Cation-Cation and Anion-Anion Replacement in Stabilizing Their Modifications. Physics of Particles and Nuclei, 46, 452-474. https://doi.org/10.1134/S106377961503003X 
[37] Earley, J.W. (1950) Description and Synthesis of the Selenide Minerals. American Mineralogist, 35, 337-364. http://www.minsocam.org/ammin/AM35/AM35 337

[38] Baikulov, R.B. and Asadov, Y.G. (2005) High-Temperature X-Ray Diffraction Study of the $\alpha \beta$ Transformation in CuAgSe. Inorganic Materials, 41, 338-342. https://doi.org/10.1007/s10789-005-0134-9

[39] Shi, C., Xi, X., Liu, E., Wu, G. and Wang, W. (2018) Vacancy Mediated Ionic Mobility in a Phonon Glass Material CuAgSe. Solid State Ionics, 326, 183-187. https://doi.org/10.1016/j.ssi.2018.10.005

[40] Setyawan, W. and Curtarolo, S. (2010) High-Throughput Electronic Band Structure Calculations: Challenges and Tools. Computational Materials Science, 49, 299-312. https://doi.org/10.1016/j.commatsci.2010.05.010

[41] Rasander, M., Bergqvist, L. and Delin, A. (2013) Density Functional Theory Study of the Electronic Structure of Fluorite $\mathrm{Cu}_{2} \mathrm{Se}$. Journal of Physics: Condensed Matter, 25, Article ID: 125503. https://doi.org/10.1088/0953-8984/25/12/125503

[42] Kashida, S., Shimosaka, W., Mori, M. and Yoshimura, D. (2003) Valence Band Photoemission Study of the Copper Chalcogenide Compounds, $\mathrm{Cu}_{2} \mathrm{~S}, \mathrm{Cu}_{2} \mathrm{Se}$ and $\mathrm{Cu}_{2}$ Te. Journal of Physics and Chemistry of Solids, 64, 2357-2363. https://doi.org/10.1016/S0022-3697(03)00272-5

[43] Liu, H., Shi, X., Xu, F., Zhang, L., Zhang, W., Chen, L., Li, Q., Uher, C., Day, T. and Snyder, G.J. (2012) Copper Ion Liquid-Like Thermoelectrics. Nature Materials, 11, 422-425. https://doi.org/10.1038/nmat3273 